\title{
BRYOPHYTA (MUSCI) DO HERBÁRIO DO JARDIM BOTÃNICO DO RIO DE JANEIRO
}

\author{
IDA DE VATTIMO - GIL \\ e \\ ITALO DE VATTIMO \\ Pesquisadores do \\ Jardim Botânico - RJ \\ Bolsistas do $\mathrm{CNPq}$
}

tiveram Organizando o Herbário de Briófitos do Jardim Botánico do Rio de Janeiro, os autores autor oportunidade de encontrar grande cópia de material identificado por V. F. Brotherus, Auflage, monografias sobre o assunto, que constam do Nat. Pflanzenfamilien de Engler-Prantl, 2 melhor, 10 e 11 Band. Dada a importância dessa coleção e com o objetivo de contribuir para um Bothor conhecimento da flora briologica brasileira e de atrair estudiosos para esse campo da du rela, até o presente relegado a um segundo plano, os autores dảo a público a primeira parte Bantaçäo de espécimes existentes no Herbário do Jardim Botảnico, envolvendo as familias ceas, Faceae, Brachytheciaceae, Bryacese, Dicranacese, Entodontaceae, Ephemeraceae, Erpodiatm trabroniaceae, Funariaceae, Hectwigiaceae e Hookeriaceae. Outras familias serß̋o relacionadas trabalho subsequiente.

\section{BARTRAMIACEAE}

1 - Breutelia dusenii Broth.

1927. Brasil - Rio de Janeiro: Serra de Friburgo, beira da estrada de ferro, M. Bandeira s.n. maio Brotherus det (RB).

2 - Breutelia frondii Hamp.

Brasil - Minas Gerais: Serra do Caraça, L. Damazio s.n. (RB).

3 - Breutelia microdonta Mitt.

Brasil - Minas Gerais: Serra de Ouro Preto, sobre rochas, 1400 msm L. Damazio s n. (RB). 4. Breutelia subtomentosa (Hamp.) Broth.

Burasil - Minas Gerais: Ouro Preto, L. Damazio 2163 (RB). Rio de Janeiro: Maus, Itatiaia,

1925 , Brothera no rio, parecendo de longe moita de Sphagnacese, M. Bandeira s.n., fevereiro Brotherus det. (RB).

- Breutelia ulei CM

Gmida Brasil - Rio de Janeiro. Granja, Estrada de Teresópolis, Friburgo, na encosta de rocha em capoeira, M. Bandeira s.n., maio 1927, det. Brotherus (RB).

Rodriguésia
Rio de Janeiro 
6 - Leiomela piligera (Hamp.) Broth.

Brasil - Rio de Janeiro: Reserva Florestal, Itatiaia, rupícola, lugar seco adiante de Maromba, Pedro Occhioni s.n., setembro 1924, Brotherus det. (RB): Maú,, Itatiaia, entre Neckeraceae e Plagiochila sp., epífita, M. Bandeira s.n., fevereiro 1925; Brotherus det. (RB).

\section{7 - Philonotis gardneri (C.M.) Broth.}

Brasil - Rio de Janeiro: Andaime Grande, Paineiras, Pedro Occhioni sn, julho 1927, Brotherus det (RB).

\section{8 - Philonotis tenella (C. M.) Broth.}

Brasil - Rio de Janeiro: Teresópolis, Estrada de Petrópolis, pedra muito molhada coberta de terra, M. Bandeira s.n., março 1926, Brotherus det. (RB): Maú, Itatiaia sobre lages de córrego na beira da estrada, M. Bandeira s.n., fevereiro 1925 (RB); Estrada das Macieiras a Montserrat, rupícola, rocha úmida na beira da estrada, M. Bandeira s.n., janeiro 1925, Brotherus det. (RB); Monnerat, Fazenda da Cachoeira, vegetando sobre barrancos em lugar seco de muita exposição, M. Bandeira s.n., abril 1928, Brotherus det. (RB). Minas Gerais: Fazenda Bom Destina. Providência, em barranco na beira da estrada, M. Bandeira s.n., março 1924 (RB); Loc. n. ind., L. Damazio s.n. (RB). Amazonas: Fonte Boa, Solimōes, J.G. Kuhlmann s.n., março 1924 (RB).

\section{9 - Bartramiaceae spp.}

Brasil - Rio de Janeiro: Serra de Itatiaia entre Macieiras e Montserrat, sobre pedra úmida (Philonotis tenella?), Gurgel s.n., juiho 1926 (RB); Pico das Agulhas Negras, Itatiaia, Príncipe de Orleans e Bragança s.n., novembro 1925 (RB); Planalto, Serra do Itatiaia, em barranco úmido na beira do estrada, M. Bandeira s.n., outubro 1925 (RB).

\section{BRACHYTHECIACEAE}

10 - Brachythecium poadelphus C.M.

Brasil - Río de Janeiro: Mauá, Itatiaia, sobre lage, em córrego, muito úmida, M. Bandeira s.n., fevereiro 1925, Brotherus det. (RB).

11 - Rhynchostegium beskeanum (C.M.) Broth.

Brasil - Rio de Janeiro: Mauá, Itatiaia, em árvore caída, beira de córrego, misturado a Pterogonium pulchellum (Hook.) Broth. (Sematophyllaceae), M. Bandeira s.n., fevereiro 1925. Brotherus det. (RB): Reserva Florestal, Itatiaia, em árvore, Pedro Occhioni s.n., dezembro 1924. Brotherus det. (RB).

\section{2 - Rhynchostegium campoidense C.M.}

Brasil - Rio de Janeiro: Monnerat, Fazenda Cachoeira, numa mata, sobre madeira em decomposiç̋̈o, M. Bandeira s.n., abril 1923, Brotherus det. (RB).

13 - Rhynchostegium rivale (Hamp.) Broth.

Brasil - Rio de Janeiro: Mauś, Itatiaia, sobre tronco em putrefaçāo, M. Bandeira s.n-n fevereiro 1925, Brotherus det. (RB).

14 - Rhynchostegium sellowii (Hornsch.) Broth.

Brasil - Rio de Janeiro: Monnerat, Fazenda Cachoeira, rupicola nas imediaçס̄es de cachoeira, em lugar muito escuro, M. Bandeira s.n., abril 1923, Brotherus det. (RB); Mouá. 
Itatiaia, sobre folhas caídas em decomposição na beira de pequena cascata, M. Bandeira s.n., fevereiro 1925, Brotherus det. (RB).

\section{BRYACEAE}

15

- Anomobryum conicum (Mont.) Broth.

(C.M. Brasil - Rio de Janeiro: Estrada das Macieiras ao Maromba, junto com Webera gramocarpa Broth., em barranco, M. Bandeira s.n., janeiro 1925, Brotherus det. (RB).

16 - Brachymenium radiculosum (Schw.) Par.

Brasil - Rio de Janeiro: mata do Registro, Serra de Friburgo, em madeira podre, M. Bandeira s.n., maio 1927. Brotherus det. (RB); Mauá, I tatiaia, sobre pau em decomposiçāo, M. Bandeira s.n., fevereiro 1925, Brotherus det. (RB): Estação Teodoro de Oliveira, Alto da Serra de Friburgo, vegetando na base das ấrvores, M. Bandeira s.n., maio 1923, Brotherus det. (RB). Minas Gerais: Pico do Itacolumi, em terras turfosas, descida ingreme, abaixo do Pico, Agnes Chase s.n., abril 1925, Brotherus det. (RB).

17 - Brachymenium hornschuchianum Mart.

Brasil - Rio de Janeiro: mata do Itatiainha, Mauá, Itatiaia, epifita, seta muito longa, muito

raro, único exemplar encontrado, M. Bandeira s.n., fevereiro 1925, Brotherus det. (RB).

18 - Bryum aberrans Hamp.

drasil - Rio de Janeiro: Fazenda da Cachoeira, Monnerat, sobre pedras, perto de uma caixa Sgua, M. Bandeira s.n., maio 1923, Brotherus det. (RB).

19 - Bryum argenteum L.

Brasil - Rio de Janeiro: cidade do Rio de Janeiro, Chácara to Lage, rua Jardim Botânico, vegetando sobre muralha fronteira, Pedro Occhioni s.n., junho 1924 (RB); ibidem, Chácara do

Lage, rua Jardim Botânico, vegetando sobre muro fronteiro, M. Bandeira s.n., agosto 1923 ,

Brotherus det. (RB).

20 - Bryum argenteum L. var. lanatum (Palis) Broth.

Brasil -Rio de Janeiro: Quebra-Frasco, Teresópolis, sobre pedra em capoeirăo, M. Bandeira 1.n., março 1926, Brotherus det. (RB): Morro Tapera, Petrópolis, em barranco, entre Polytrichaceae, M. Bandeira s.n., abril 1924, Brotherus det. (RB); Mauá, Itatiaia, sobre rochas áridas, próximo à mata do Rio Preto, M. Bandeira s.n., fevereiro 1925, Brotherus det. (RB).

21 - Bryum argenteum L. var. majus Bryol. Eur.

Prasil -Rio de Janeiro: Mauá, I tatiaia, em terra cobrindo rochas úmidas, M. Bandeira s.n., tvereiro 1925, A. Grout e R. S. Williams det. (RB).

22 - Bryum argenteum L. var. robustum Broth.

1922 Brasil - Rio de Janeiro: Pico das Agulhas Negras, Itatiaia, J.G. Kuhlmann s.n., outubro

1922. Brotherus det (RB); Pieo das Agulhas Negras, Itatiaia, em rochas, M. Bandeira s.n., janeiro 925 (RB).

23 - Bryum densifolium Brid.

Occhionil - Pio de Janeiro: cidade do Rio de Janeiro: Andaime Grande, Paineiras, Pedro ioni s.n., julho 1927. Brothorus det. (RB). 
Brasil - Minas Gerais: Serra de Antonio Pereira, L. Damazio 2118 (RB).

25 - Pohlia sp.

Brasil - Rio de Janeiro: Itatiaia, Alto do Itatiaia, J.G. Kuhimann, s.n., outubro 1922, M. Bandeira det. (RB).

26 - Bryaceae spp.

Brasil - Rio de Janeiro: caminho dos Trés Picos, Serra do Itatiaia, na mata em árvore viva, Bandeira s.n., outubro 1926 (RB); Macieiras, Serra do Itatiaia, local úmido sobre barranco, Gurgel s.n., julho 1925 (RB); Mauá, em tijolos, na beira da estrada, M. Bandeira s.n., fevereiro 1925 (RB); Planalto, Serra do Itatiaia, nas fendas de rochas úmidas, M. Bandeira s.n., outubro 1926 (RB): Planalto, Serra do Itatiaia, em terra turfosa, M. Bandeira s.n., outubro 1926 (RB); Macieiras, Serra do Itatiaia, sobre pedra, Gurgel s.n., julho 1926 (RB); Planalto, Serra do I tatiaia, nas fendas de rochas, M. Bandeira s.n., outubro 1926 (RB); Planalto, Serra do Itatiaia, nas fissuras úmidas das rochas, M. Bandeira s.n., outubro 1926 (RB).

\section{DICRANACEAE}

27 - Campylopus arenicola (C.M.) Mitt.

Brasil - Rio de Janeiro: cidade do Rio de Janeiro: Andaime, Ponte do Inferno, Corcovado, entre esfagno, M. Bandeira s.n., março 1925, Grout. det. (RB).

28 - Campylopus beyrichii Dub.

Brasil - Rio de Janeiro: cidade do Rio de Janeiro; Macieiras, Itatiaia, em terra turfosa e úmida, M. Bandeira s.n., janeiro 1925, Brotherus det, (RB).

29 - Campylopus filifolius (Hornsch.) Mitt.

Brasil - Rio de Janeiro: Fazenda Cachoeira, Monnerat, sobre troncos em decomposiçāo, abril 1923, Brotherus det. (RB); Chapadão do Quebra-Frasco, Teresópolis, em capoeirão, sobre tronco em decomposiçāo, lugar de muita sombra, M. Bandeira s.n., Vattimo-Gil det. (RB).

30 - Campylopus occhionii Broth.

Brasil - Rio de Janeiro: cidade do Rio de Janeiro, local úmido, esteril, Pedro Occhioni s.n., agosto 1924, Brotherus det. (RB).

31 - Campylopus penicillatus (Hornsch.) Broth.

Brasil - Rio de Janeiro: Reserva Florestal, Itatiala, rupicola, próximo â ponte do Maromba, lugar seco, Pedro Occhioni s.n., setembro 1924, Brotherus det. (RB).

32 - Dicranella guilleminiana (Mont.) Broth.

Brasil - Rio de Janeiro: Maus, Itatiaia, em terra cobrindo rocha úmida, M. Bandeira s.n., fevereiro 1925, Brotherus det. (RB).

33 - Dicranella hilariana (Mont $p$, ) Broth

Brasil - Rio de Janeiro: Estrada de Petrópolis, em Teresópolis, sobre saibro, barranco, beira do estrada, lugar seco, M. Bandeira s.n. (RB); Poço d'Antas, Teresópolis, beira da mata, em 
barranco, lugar de exposição. M. Bandeira s.n., março 1926 (RB); Morro da Tapera, Petrópolis, em barranco, Bandeira s.n., abril, Brotherus det. (RB).

- Dicranella martiana Hamp.

Brasil - Rio de Janeiro: Estrada das Macieiras a Montserrat, Itatiaia, em barranco, M. Bandeira s.n., janeiro 1925 (RB); Cidade do Rio de Janeiro, Pico do Corcovado, junto com Dicranella guilleminiana (Mart.) Broth., em barranco úmido de barro vermelho, M. Bandeira s.n., janeiro 1925, Brotherus det. (RB).

35 - Dieranodontium (Bryol. eur. fasc. 41) sp.

Brasil - Rio de Janeiro: Fazenda da Cachoeira, Monnerat, dentro de uma mata, num brejo, M. Bandeira s.n., abril 1923, Bandeira det. (RB).

36 - Dicranum Hedw. sp.

Brasil - Amazonas: Manaus, em terreno arenoso, fragrantissimo, ex Herb. Schwacke 4161 (III, 581), julho 1882 (RB).

37 - Dicranum Hedw. sp.

Brasil - Rio de Janeiro: Mauá, ex Herb. Schwacke 909, ano 1875 (RB).

38 - Holomitrium crispulum Mart.

Brasil - Rio de Janeiro: Estrada de Petrópolis, em Teresópolis, misturado com Pilopogon subjulaceus Hamp., sobre argila cobrindo pedra, beira de estrada, lugar de exposiçăo, M. Bandeira s.n., Brotherus det. (RB); Mauá, Itatiaia, na mata das cabeceiras do nio Preto, sobre pau em decomposiç̧̃o, M. Bandeira s.n., fevereiro 1925, Brotherus det. (RB).

39 - Holomitrium Brid. sp. det. (RB).

Brasil - São Paulo: Campo Grande, Alto da Serra, Kuhlmann s.n., outubro 1922, Brotherus 40 - Pilopogon subjulaceus Hamp.

Brasil - Rio de Janeiro: Alto da Serra, Petrópolis, em um barranco úmido, M. Bandeira s.n., fevereiro 1924, Brotherus det. (RB); Estrada Petrópolis, em Petrópolis, sobre argila cobrindo pedra beira da estrada lugar de exposiçä́o, M. Bandeira s.n, março 1926. Brotherus det (RB); Estrada das Macieiras ao Maromba, Itatiaia, em barranco, M. Bandeira s.n., Brotherus det. (RB).

\section{1 - Trematodon reflexus C.M}

Brasil - São Paulo: Piassoguera, Santos, vegetando num barranco, do rio, J.G. Kuhlmann s.n., outubro 1922, Brotherus det. (RB). Rio de Janeiro: Alto da Serra, Petrópolis, em barranco úmido, M. Bandeira s.n., A. Grout e V. F. Brotherus det. (RB); Poço das Antas, Teresópolis, em barrancos, lugar de exposição na beira da mata, M. Bandeira s.n., março 1926, Brotherus det. (RB).

42 - Trematodon Michx. sp.

Brasil - Rio de Janeiro: Alto da Serra, Petrópolis, sobre argila em exposiç̄̄o, barranco vertical, Agnes Chase s.n., abril 1925 (RB).

43 - Trematodon Michx. sp. 
Brasil - Rio de Janeiro: Estrada de Teresópolis, Friburgo, em barranco, na beira da estrada, M. Bandeira s.n., maio 1927 (RB).

44 - Thysanomitrium richardi Schw.

Brasil - Rio de Janeiro: Maú, Itatiaia, em barranco, na sede do Núcleo Visconde de Mauá, M. Bandeira s.n., Brotherus det. (RB).

\section{5 - Dicranaceae spp.}

Brasil - Rio de Janeiro: Estrada de Petrópolis, em Teresópolis, barranco de pedra, na beira da estrada, lugar de exposiçăo, M. Bandeira s.n., (RB). Estrada das Macieiras ao Maromba, Itatiaia, em barranco, M. Bandeira s.n., janeiro 1925 (RB); Montserrat, Serra do Itatiaia, vegetando sobre tronco em decomposiçăo, Gurgel s.n., julho 1926 (RB); Teodoro de Oliveira, Serra de Friburgo, em barranco, M. Bandeira s.n., maio 1927 (RB); Macieiras, Serra do Itatiaia, sobre barranco, Gurgel s.n., julho 1926 (RB); Granja, Estrada de Teresopolis, em Friburgo, em madeira podre, M. Bandeira s.n., maio 1927 (RB).

\section{ENTODONTACEAE}

46 - Entodon splendidus Hamp.

Brasil - Rio de Janeiro: Monnerat, Fazenda Cachoeira, saxícola, vegetando perto de uma cascata, M. Bandeira s.n., abril 1923, Brotherus det (RB).

\section{7 - Entodon splendidulus Hamp}

Brasil - Rio de Janeiro: Chapad̋̃o Quebra-Frasco, Teresópolis, na sombra, em capoeirão, em tronco vivo, M. Bandeira s.n., março 1926, Brotherus det. (RB); Chapadß̋o Quebra-Frasco, Teresópolis, sobre tronco em decomposiçăo, lugar de muita sombra, M. Bandeira s.n., março 1926, Brotherus det. (RB).

\section{8 - Entodon polysectus C.M}

Brasil - Rio de Janeiro: Macieiras, Itatiaia sobre tronco de Melastomataceae, M. Bandeira s.n., janeiro 1925, Brotherus det. (RB).

49 - Erythrodontium longisetum (Hook.) Broth.

Brasil - Rio de Janeiro: Poço d'Antas, Teresópolis, na mata virgem, lugar de sombra, sobre tronco vivo, M. Bandeira s.n., março 1926, Brotherus det. (RB); Praça D. Afonso, Petropolis, cortícola, M. Bandeira s.n., fevereiro 1924, Brotherus det. (RB).

50 - Erythrodontium squarrosum (C. M.) Broth.

Brasil - Minas Gerais: Fazenda Bom Destina, Providència, misturado com Papillaria appressa (Hornsch.) Broth., epifita em tronco de flambosia, M. Bandeira s.n., março 1924, Brotherus det. (RB). Rio de Janeiro: Monnerat, Fazenda Cachoeira, misturado com Papillaria appressa eplfita vege tando a beira da estrada em lugar seco e de muita exposiçāo, M Bandeira s.n., abril 1923, Brotherus $\operatorname{det}$ (RB).

\section{1 - Entodontacese sp.} 1925 (RB).

Brasil - Rio de Janeiro: Macieiras, Serra do Itatiaia, em lenho podre, Gurgel s.n., julho 


\section{EPHEMERACEAE}

52 - Ephemerum Hamp. sp.

(RB).

Brasil - Santa Catarina: Itajal, F. Mueller 5814, janeiro 1882, ex Herb. Schwacke 5814

\section{ERPODIACEAE}

53 - Erpodium sp.

Brasil - Rio de Janeiro: cidade do Rio de Janeiro, Jardim Botânico, no Tamarindus indica, em frente à secretaria, M. Bandeira s.n., outubro 1923 (RB).

\section{FABRONIACEAE}

54 - Fabronia subpolycarpa C. M.

Brasil - Minas Gerais: Bom Destino, Providência, corticola, na mata, M. Bandeira s.n., março 1924, Brotherus det. (RB).

\section{FUNARIACEAE}

55 - Physcomitrium angustifolium Broth.

Brasil - Rio de Janeiro: cidade do Rio de Janeiro, Jardim Botânico, viveiros, vegetando entre Bauhinias em germinaçäo, M. Bandeira s.n., setembro 1923, Brotherus det. (RB, tipo).

56 - Physcomitrium (Brid.) Fuernr. sp.

Brasil - Rio de Janeiro: cidade do Rio de Janeiro, Jardim Botånico, em sementeira de Mimosa pudica, foram observadas palhetas cintilantes, M. Bandeira s.n., outubro 1927 (RB).

\section{HEDWIGIACEAE}

57 - Harrisonia humboldtii Spreng.

Brasil - Minas Gerais: Itacolumi, em rochas a 1600 msm, L Damazio s.n., (RB).

\section{HOOKERIACEAE}

58 - Callicostella microcarpa (Hornsch.) Broth.

Brasil - Rio de Janeiro: Mata do Registro, Serra de Friburgo, em lenho podre, M. Bandeira s.n., maio 1927 (RB).

59 - Callicostella pallida (Hook.) Jacq.

Brasil - Rio de Janeiro: Monnerat, Fazenda Cachoeira, num œorrego sobre folhas a outros materiais em decomposiç̋̆o, M. Bandeira s.n., abril 1923, Brotherus det. (RB).

60 - Callicostella paulensis (C. M.) Broth.

Brasil - Minas Gerais: Fazenda Bom Destina, Providència em tronco em decomposiçăa M Bandeira s.n., março 1924, Brotherus det. (RB). Rio de Janeiro: Antiga estrada do Alto da Serra de Friburgo, vegetando sobre tronco caído, M. Bandeira s.n., março 1925, Brotherus det. (RB). 
Brasil - Rio de Janeiro: Mauá, Itatiaia, sobre tronco caído, beira de picada na mata, M. Bandeira s.n., fevereiro 1925, Brotherus det. (RB).

62 - Cyclodictyon olfersianum (Hornsch.) Broth.

Brasil - Minas Gerais: Fazenda Bom Destina. Providência, misturado com Vesicularia glaucopinnata C. M., em tronco em decomposição na mata, M. Bandeira s.n., março 1924, Brotherus det (RB); Fazenda Bom Destina, Providència rupicola misturado com Glossadelphus truncatus (C. M.) Broth., sobre pedras muito úmidas na mata, M. Bandeira s.n., março 1924, Brotherus det. (RB); Fazenda Bom Destino, Providência, em tronco ęm decomposição, mata, M. Bandeira s.n., março 1924 (RB). Rio de Janeiro: mata do Registro, Serra de Friburgo, em rochas úmidas, M. Bandeira s.n., maio 1927, Brotherus det. (RB).

63 - Helicodontium capillare (Sw.) Broth.

Brasil - Rio de Janeiro: Chapadß̊o de Quebra-Frasco, Teresópolis, em capoeirão, lugar sombrio, em cipó em decomposição, M. Bandeira s.n., marழ̣o 1925, Brotherus det. (RB).

64 - Hookeriopsis asperella Hamp.

Brasil - Rio de Janeiro: Reserva Florestal, Itatiaia, em pau em decomposição, próximo à cachoeira Maromba, Pedro Occhioni s.n., setembro 1924, Brotherus det. (RB).

65 - Hookeriopsis beyrichiana (Hamp.) Broth.

Brasil - Rio de Janeiro: Ponte do Inferno, Corcovado, rupicola, na sombra, úmído, M. Bandeira s.n., fevereiro 1925, Brotherus det. (RB).

66 - Hookeriopsis drepanophylla (Geg. et Hamp.) Broth.

Brasil - Rio de Janeiro: cachoeira do Maromba, Serra do Itatiaia, sobre rocha, Pedro Occhioni s.n., maio 1927, Brotherus det. (RB).

67 - Hookeriopsis incurva (Hook. et Gres.) Broth.

Brasil - São Paulo: Estaçlo Biológica, J. G. Kuhlmann s.n., outubro 1922, M. Bandeira e Grout-Williams det. (RB). Rio de Janeiro: Reserva Florestal, Itatiaia, sobre pau em decomposição, Pedro Occhioni s.n., dezembro 1924, Bandeira, Grout-Williams e Brotherus det. (RB); Morro da Tapera, Petrópolis, M. Bandeira s.n., abril 1924 (RB); Monnerat, Fazenda Cachoeira, sobre substâncias em decomposiç̋̃o à beira de cascata, M. Bandeira s.n., Brotherus det. (RB); Mata do Registro, Serra de Friburgo, em madeira podre, M. Bandeira s.n., Brotherus det. (RB).

68 - Hookeriopsis rubens (C. M.) Broth.

Brasil - Rio de Janeiro: Granja, Estrada de Teresópolis, Friburgo, cm madeira podre, M. Bandeira s.n., maio 1927, Brotherus det. (RB).

69 - Lepidopilum brevisetum (Hamp.) Broth.

Brasil - Rio do Janeiro: Mauá. Itatiaia, epifita, na mata, M. Bandeira s.n., fevereiro 1925. Brotherus det. (RB).

70 - Lepidopilum monilodontium Hamp.

Brasil - Rio de Janeiro: Pedreira da Quitandinha, Petrópolis, epifita, M. Bandeira s.n., fevereiro 1924, Brotherus det. (RB); Monnerat, Fazenda Cachoeira, epifita, M. Bandeira s.n., abril 
1923, Brotherus det. (RB): Pedreira de Quitandinha, Petrópolis, rupícola, local muito úmido, Bandeira s.n., fevereiro 1924, Brotherus det. (RB); Estação Teodoro de Oliveira, Alto da Serra de Friburgo, epifita, M. Bandeira s.n., maio 1923, Brotherus det. (RB): Reserva Florestal, Itatiaia, em árvore, Pedro Occhioni s.n., dezembro 1924, Brotherus det. (RB). Minas Gerais: Serra da Grama, misturado com /sopterygium curvicollum (C. M.) Broth., em tronco de ârvore, descida ingreme da mata, 1000 msm, Agnes Chase s.n., abril 1925, Brotherus det. (RB).

71 - Lepidopilum subfuscum Mitt.

Brasil - Minas Gerais: Fazenda Bom Destina Providència em tronco, na mata M Bandeira s.n., março 1924, Brotherus det. (RB).

72 - Philophyllum tenuifolium (Mitt.) Broth.

Brasil - São Paulo: Estaçāo Biológica, Campo Grande, Alto da Serra, epifita, em bromeliácea, Kuhimann s.n., outubro 1922, Brotherus det. (RB).

\section{3 - Hookeriaceae spp.}

Brasil - Rio de Janeiro: Cachoeira do Maromba, Itatiaia, sobre terra cobrindo pedra, M. Bandeira s.n., (RB); Mauá, Itatiaia, verde clara, sobre pau em decomposiçāo, M. Bandeira s.n., fevereiro 1925 (RB).

\section{ABSTRACT}

The Authors give the first part of the catalogue of the Bryophyta (Musci) of the Jardim Botânico do Rio de Janeiro Herbarium. Seventy three specimens identified by V. F. Brotherus are listed, belonging to the families Bartramiaceae, Brachytheciaceve, Bryacese, Dicranaceae, Entodontaceae, Ephemeraceae, Erpodiacese, Fabroniaceae, Funariaceae, Hedwigiacese and Hookeriacese.

\section{LITERATURA CONSULTADA}

V. F. Brotherus - Nouvelles Contributions a la Flore Bryologique du Brésil, Bih K. Sv. Wet Ak. Handl., Bd 21, Afd III, 1895.

- Musci in Pflanzenfam 2 Auflage, 10 band, 1 Haelfte: 143-478 1924

Musci, 2 Haelfte, in Pflanzenfam. 2 Auflage, 11 Band: 1-522, 1925.

Hampe, E. - Symbolae ad floram Brasiliae centrales cognoscendam, in Vidensk. Meddel. Naturh. Foren. Kjobenhavn: 72, 74, 77, 1870.

Mitten, W. - Musci austro-americani, in Journ. Linn. Soc. XII, 1869.

Mueller, C. - Bryologia Serrae Itatiaiae, in Bull. I'Herb. Boiss. VI.

Ule, E. - Die Verbreitung der Torfmoose in Brasilien, Englers Bot. Jahrb., 1899. 